\title{
Phonocardiographic method of assessing changes in left ventricular function after Starr-Edwards replacement of aortic valve
}

\author{
D. G. Gibson, G. Broder, and E. Sowton \\ From the National Heart Hospital and Institute of Cardiology, London W.I
}

A phonocardiographic method is described for measuring the time taken by the ball of a StarrEdwards prosthesis to move to the apex of the cage at the onset of left ventricular ejection, and from this its acceleration can be derived. The acceleration is conspicuously increased in post-ectopic beats and in exercise, but is unaffected by posture and tachycardia induced by atropine or ventricular pacing. It is suggested that the acceleration of the ball is related to the initial acceleration of blood into the ascending aorta.

In the experimental animal, peak acceleration of blood into the ascending aorta has proved to be a sensitive measure of left ventricular function (Rushmer, 1964; Noble, Trenchard, and Guz, I966a, b, c), reflecting the initial impulse generated by the development of tension in the myocardium (Franklin, Van Citters, and Rushmer, 1962). It was thought that the initial movement of the ball of a StarrEdwards prosthesis in the aortic position would also show such a relation. A phonocardiographic method assessing ball movement was therefore devised and its use as a test of left ventricular function explored.

\section{Methods and subjects}

Theory The early systolic sound from an aortic prosthesis $\left(A_{1}\right)$ is delayed relative to the onset of left ventricular ejection by the time interval necessary for the ball to travel from one end of the cage to the other (Fig. Ia). Since the closure sound from the prosthesis $\left(A_{2}\right)$ occurs at the end of left ventricular ejection, the difference between the $A_{1} A_{2}$ interval and the ejection time represents the ball travel time. This is not affected by the transmission time of the aortic pressure measuring system, since the incisuras at the beginning and end of ejection are delayed by equal amounts.

The acceleration undergone by the ball can be calculated if the dimensions of the prosthesis are known, since $s=u t+\frac{1}{2} a t^{2}$ where $s=$ the distance travelled by the ball, $u=$ the initial velocity of the ball (zero), $t=$ the ball travel time, and $a=$ the acceleration undergone by the ball.

Received 4 July 1969.
The maximum velocity is given by $v=a t$, assuming that peak velocity is reached just before the ball strikes the apex of the cage.

Studies were made of 42 patients who had had Starr-Edwards prostheses inserted in the aortic position at the National Heart Hospital I to 36 months previously. Of these, 5 had, in addition, mitral valve prostheses. Maintenance digitalis, diuretics, and anticoagulant therapy were continued during the period of study.

Haemodynamic studies were performed on 6 patients, 4 in sinus rhythm and 2 in atrial fibrillation, who had been referred for investigation on account of an early diastolic murmur associated with evidence of reduced red cell survival. Aortography revealed trivial aortic regurgitation in all except one patient (Case 6) in whom it was sufficiently severe to require replacement of the prosthesis. A No. 8 Teflon catheter was inserted by the Seldinger technique and advanced to the aortic root. A No. 8 twin-lumen catheter was introduced through an arm vein, and advanced to the wedge pulmonary artery position. Phonocardiograms were recorded from the lower left sternal edge with a Sanborn microphone, and pressures were measured with Statham $\mathrm{P}_{23} \mathrm{~Gb}$ strain gauge transducers, with a simultaneous electrocardiogram. Pressure traces, electrocardiogram, and phonocardiogram were recorded photographically at a paper speed of $100 \mathrm{~mm}$./sec. Cardiac output was measured by the dye dilution technique, $5 \mathrm{mg}$. indocyanine green being injected into the main pulmonary artery and blood withdrawn from the aortic root by a Kipp and Zonen constant rate pump through a Gilford cuvette. Cardiac outputs, pressures, and phonocardiograms were recorded at rest and after 2 minutes of exercise at $100-200 \mathrm{kpm} . / \mathrm{min}$. 
In the other 36 patients, all in sinus rhythm, electrocardiograms and phonocardiograms were recorded, using a Cambridge photographic recorder, at a paper speed of $100 \mathrm{~mm}$./sec. Seven patients were exercised erect on a bicycle ergometer, and records were made at increasing work loads. No attempt was made to reach the exercise tolerance of the patients. Seventeen patients were exercised supine, with 2 minutes straight leg raising; a recording was made during exercise and in the recovery period until the heart rate had returned to the resting level. Atropine, $0.6 \mathrm{mg}$., was administered to 6 patients after control recordings and recordings during supine exercise had been made. The heart rate was allowed to return to the previous level before the injection of atropine was given, and it was preceded by a control injection of an equal volume of saline. The effect of posture was investigated in 10 patients. Recordings were made with the patients supine, sitting, and standing. The effects of ventricular pacing were observed in 3 patients. In 2 , in the immediate post-operative period, an external pacemaker was used, and I had an implanted ventricularly inhibited unit. In all, epicardial leads were implanted in the right ventricle. In 13 patients ectopic beats occurred spontaneously at rest. The effect on the $\mathrm{QA}_{1}$ intervals of the immediately succeeding beats was observed.

The timing of heart sounds on the phonocardiograms was measured from the onset of the first high frequency component. Since the $Q$ wave on the electrocardiogram cannot be identified precisely on exercise, the $\mathrm{QA}_{1}$ interval was measured from the peak of the $R$ or $S$ wave and to this was added the $Q R$ or $Q S$ interval determined from the mean of Io measurements at rest. Ejection time was measured as the interval between the mid-points of the incisuras at the beginning and end of left ventricular ejection.

\section{Results}

Ball travel time The results of simultaneous phonocardiographic and haemodynamic measurements are given in the Table. The left ventricular ejection time was always longer than the $A_{1} A_{2}$ interval, showing that the ball travel time could be consistently measured. Resting values of ball travel time were in the range $27-55 \mathrm{msec}$. in patients in sinus rhythm, and 10-60 msec. in 2 patients with atrial fibrillation. The corresponding values of maximum velocity were $28-54 \mathrm{~cm}$./ sec. and 30-120 msec., and of acceleration $0.5-1.9 g$ and $0.5-12 g$. During exercise there was little change in stroke volume; left ventricular ejection time was reduced, with corresponding increase in mean systolic ejection rate. Associated with this there was a reduction in ball travel time and an increase in acceleration by up to 8 times the resting value. The end-diastolic gradient across the prosthesis $(\Delta \mathrm{P})$, the difference between enddiastolic aortic and pulmonary capillary pres-

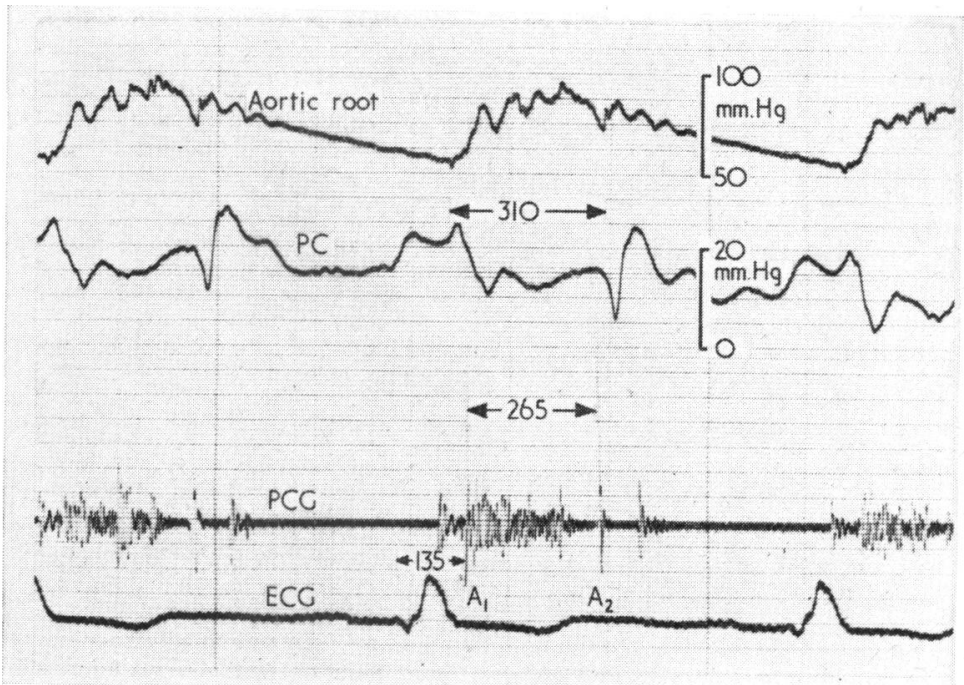

FIG. Ia Simultaneous recording of aortic root pressure, phonocardiogram, and electrocardiogram. The ball travel time is $45 \mathrm{msec}$., the difference between the $A_{1} A_{2}$ interval and the ejection time. In this patient, the mitral valve has also been replaced.

FIG. Ib Phonocardiogram recorded at rest and during erect exercise at $300 \mathrm{kpm} . / \mathrm{min}$. showing reduction in the $Q A_{1}$ interval from 170 to $130 \mathrm{msec}$.

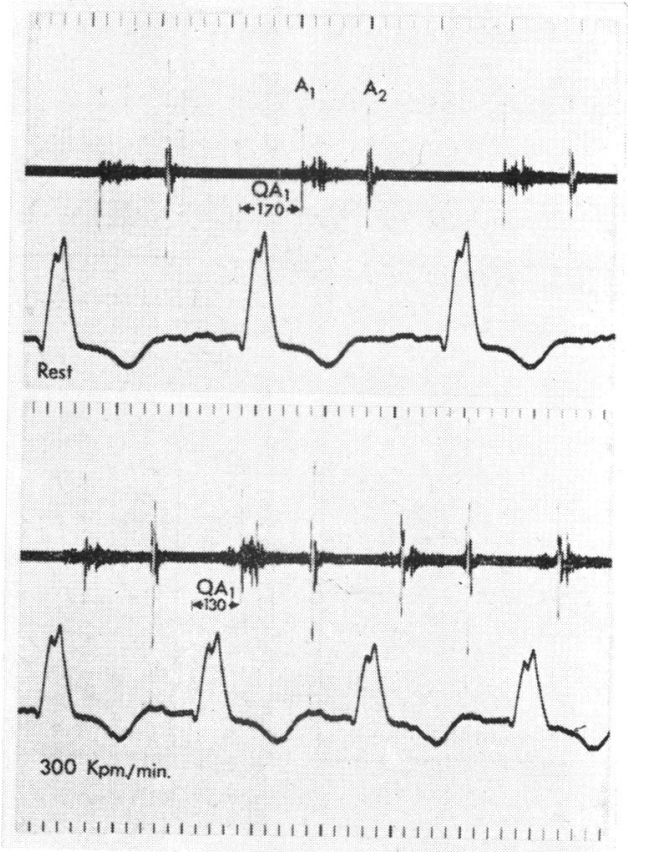


TABLE Haemodynamic values in six patients

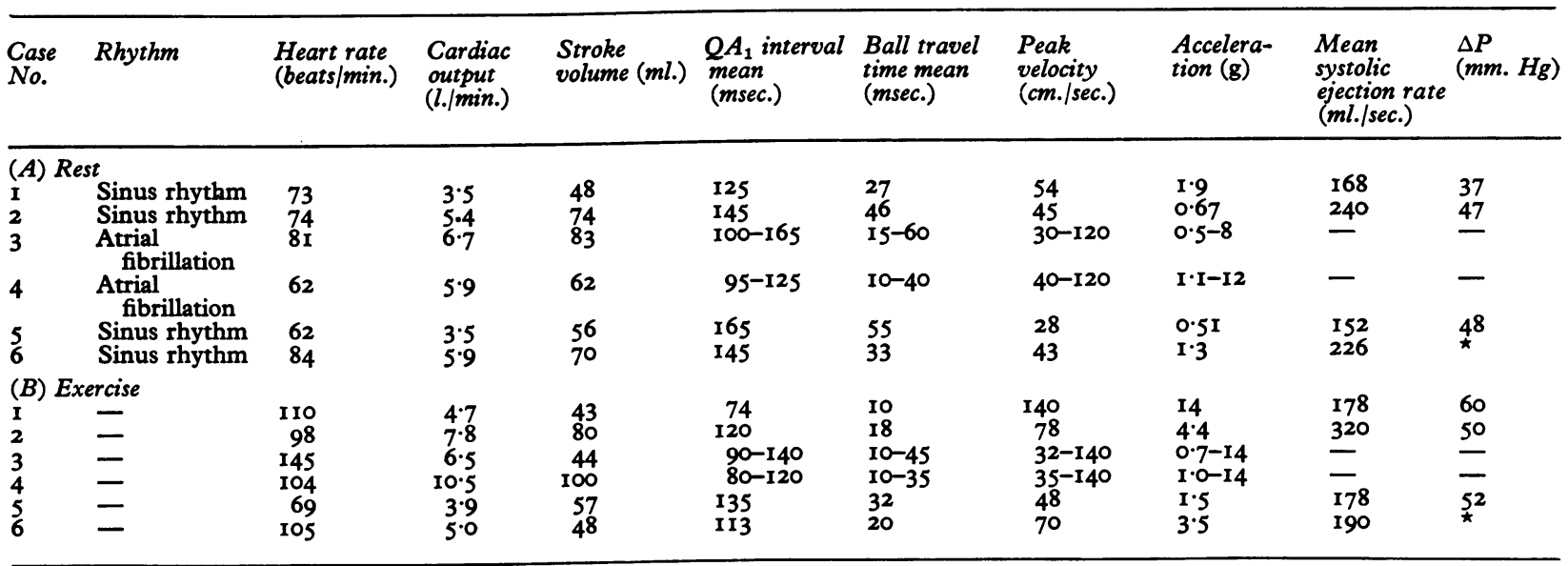

$\star$ Satisfactory wedge not obtained. $\Delta P=$ End-diastolic gradient across the prosthesis.

sures, also increased with exercise in 3 patients in whom it was measured.

Relation between travel time and $\mathbf{Q A}_{1}$ interval In all 6 patients there was a linear relation between $\mathrm{QA}$ interval and ball travel time (Fig. 2). The slope of the regression line varied between patients in the range 0.55 to 0.9.

Effect of exercise Erect exercise caused a progressive reduction in the $Q A_{1}$ interval as the heart rate increased. A typical response is shown in Fig. 3a, and similar results were obtained in a further 6 patients studied during erect exercise and in 17 patients in supine exercise (Fig. 3b). During the recovery period, there was progressive lengthening of the $\mathrm{QA}_{1}$

FIG. 2 Relation between $Q A_{1}$ interval and ball travel time in Case 1 .

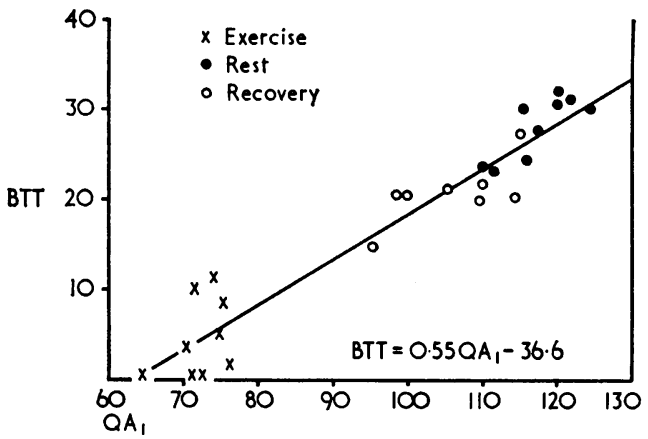

FIG. 3a Relation between the $Q A_{1}$ interval and heart rate during exercise at increasing loads in a single patient. Each point represents $a$ heart beat.

FIG. 3b Relation between the $Q A_{1}$ interval and heart rate at rest and during supine exercise in 17 patients. Each point represents the mean of 10 beats.
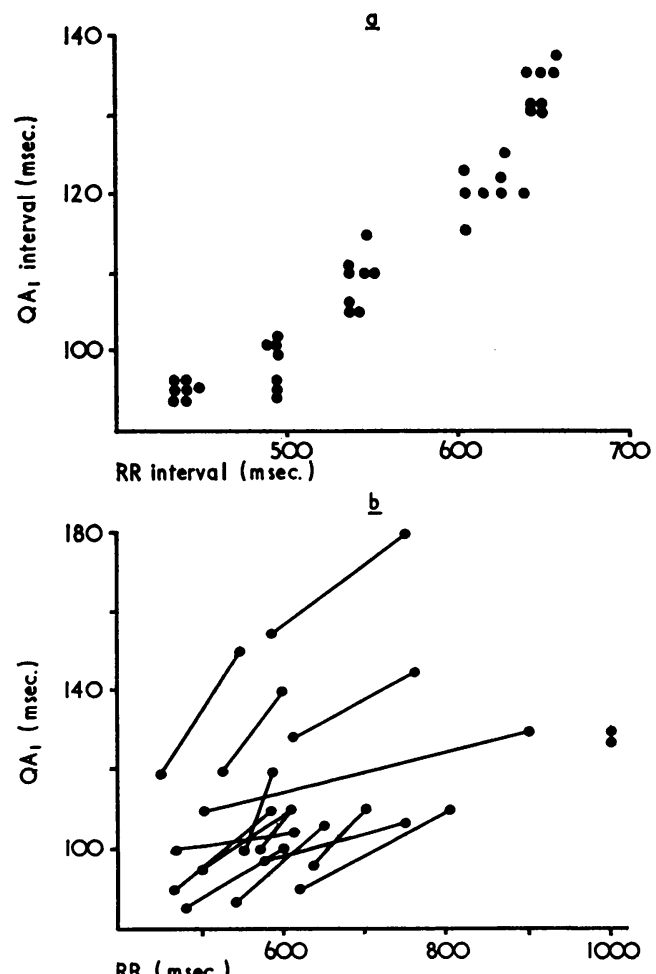
interval as the heart rate slowed. In I patient there was no change in either heart rate or $\mathbf{Q A}_{1}$ interval with exercise.

Effect of atropine The control injection of I ml. saline had no effect, but after atropine there was an increase in heart rate, with either no change or a slight increase in $\mathbf{Q A}_{1}$ interval. Typical results are shown in Fig. $4 \mathrm{a}$ and $4 \mathrm{~b}$. The relation between heart rate and $\mathrm{QA}_{1}$ thus differed from that during exercise.

Effect of posture In Io patients, movement from supine to erect position was not associated with significant change in the $\mathrm{QA}_{1}$ interval. However, there was a significant reduction in the $A_{1} A_{2}$ interval ( $\left.p<0.001\right)$ which, in the absence of any change in the $\mathrm{QA}_{1}$ interval, suggests that the ejection time was reduced (Fig. 5).

FIG. 4a Comparison of the effects of exercise and atropine-induced tachycardia on the $Q A_{1}$ interval in a single patient.

FIG. 4b Effects of atropine and supine exercise in 5 patients. Each point represents the mean of 10 beats; the range is also indicated.
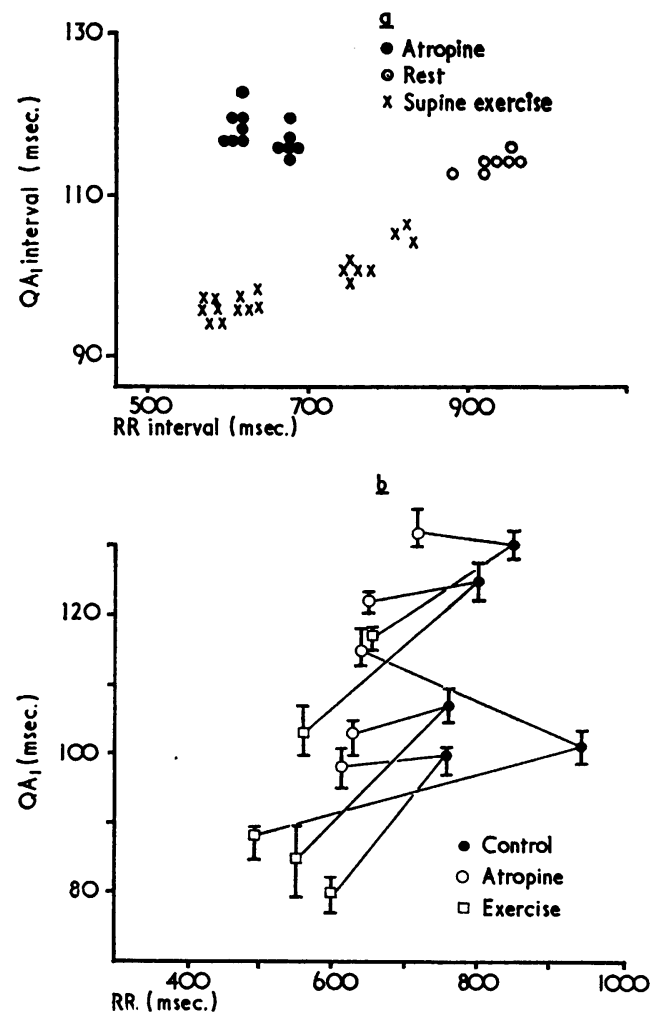

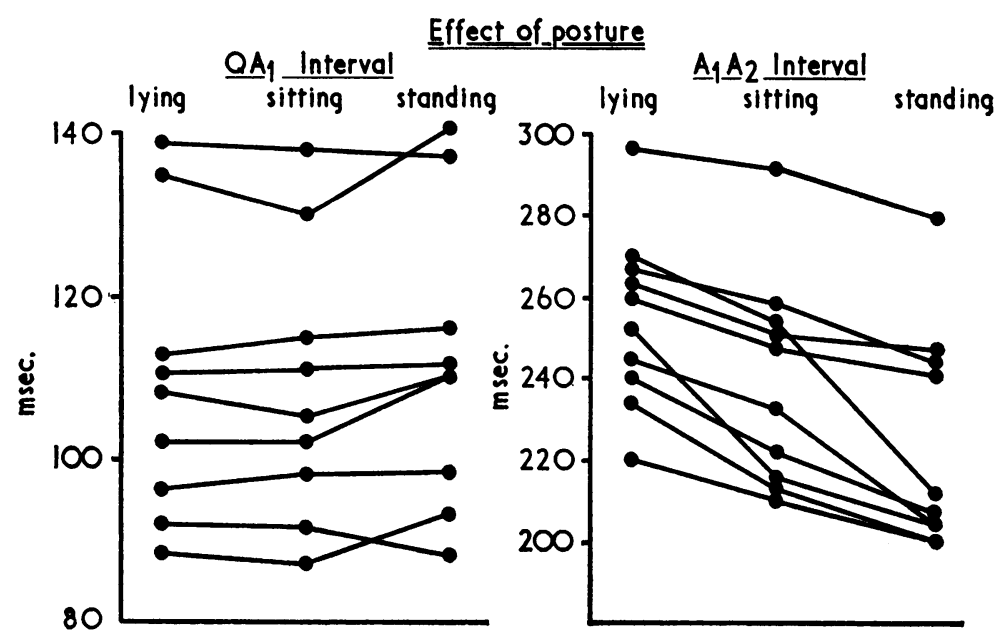

FIG. 5 Effect of posture on $Q A_{1}$ and $A_{1} A_{2}$ intervals in 10 patients. Each point represents the mean of 10 beats.

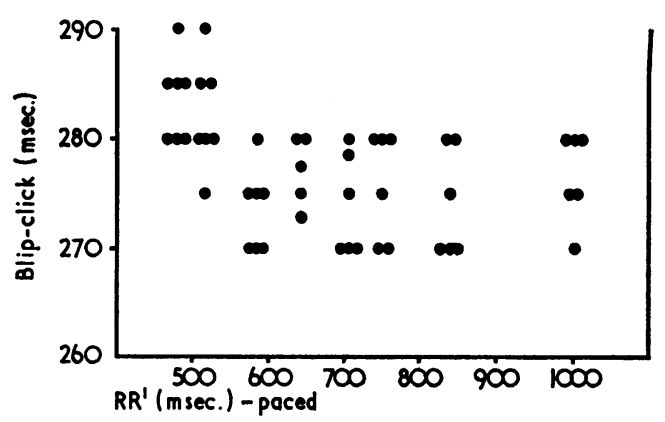

FIG. 6 Relation between pacing rate and the interval between the pacing stimulus (Blip) and $A_{1}$ (Click) in a single patient with an external pacemaker. Each point represents one beat.

Ventricular pacing There was little change in $\mathrm{QA}_{1}$ with increased frequency of pacing (Fig. 6). However, in I patient, when the heart rate was held constant at 72 beats/min., shortening of the $\mathrm{QA}_{1}$ interval could be shown immediately after exercise (Fig. 7).

Ectopic beats Ball travel time and $Q_{A_{1}}$ were reduced in all post-ectopic beats recorded. In the second beat after an ectopic $\mathbf{Q A}_{1}$ was significantly longer than control (Fig. 8a), and alternation persisted for several beats. Ball travel time was measured for the ectopic beat itself on 2 occasions, and in both it was 


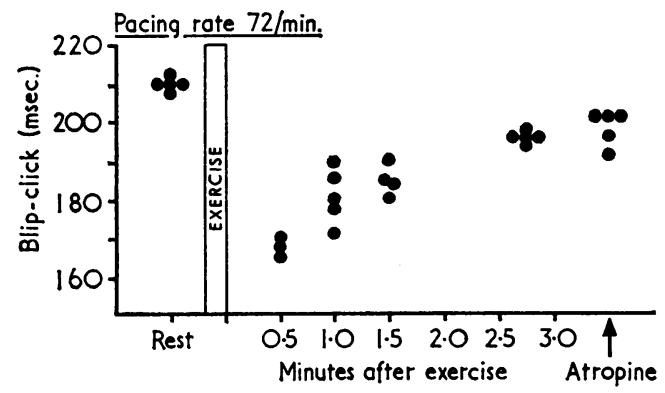

FIG. 7 Effect of exercise in a single patient with an implanted pacemaker, in whom the heart rate was held constant at 72 beats/min. Each point represents one beat.

prolonged. Associated with the ectopic beat there was an increase in the end-diastolic gradient across the prosthesis, while with postectopic beats this was decreased (Fig. 8b).

\section{Discussion}

The mechanism of production of the 2 main sounds from an aortic Starr-Edwards prosthesis is well established. Both in vivo and in vitro in a pulse duplicator, Dayem and Raftery (1967) showed that the early systolic sound was due to the ball impinging on the apex of the cage, and was thus delayed relative to the onset of left ventricular ejection. The late systolic sound was due to the ball returning to the ring, and therefore coincided exactly with the end of left ventricular ejection. The peak velocity of the ball is of the same order at the beginning and end of systole (Gimenez et al., 1965), and therefore the sounds are of similar amplitude. Dayem and Raftery also described a series of low frequency vibrations after mitral valve closure which coincided with dislodgement of the ball from the ring at the start of left ventricular ejection: if these could be recognized and identified consistently, the information necessary for measurement of ball travel time would be present on a single phonocardiogram. Since this was not the case, we have calculated ball travel time as the difference between the left ventricular ejection time measured from a central aortic pressure pulse, and the interval between the 2 main aortic sounds. The sounds are of higher frequency and greater amplitude than normal heart sounds and can therefore be recorded under a variety of conditions, including exercise (Fig. Ib), and the immediate post-operative period. The aortic pressure pulse has well-defined incisuras at the beginning and end of left ventricular ejection, which facilitate measure-
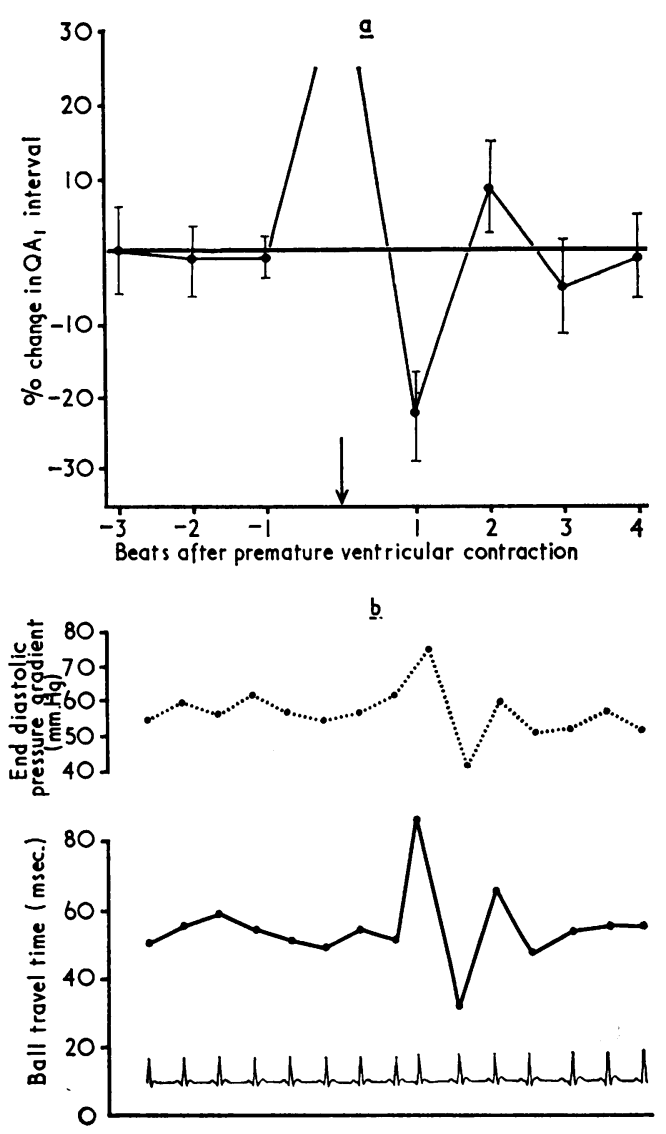

FIG. 8a Effect of premature ventricular contractions on the $Q A_{1}$ interval: mean results for 13 patients. Alterations in $Q A_{1}$ are expressed as percentage change from control (100\%) and standard deviations are also shown. Since activation is abnormal for the premature ventricular complex, $Q A_{1}$ cannot be measured.

FIG. 8b Effect of a premature supraventricular contraction on ball travel time and enddiastolic gradient across the prosthesis in a single patient (Case 5).

ment of ejection time (Fig. ra). The delay in $A_{1}$ relative to the onset of left ventricular ejection means that $A_{1} A_{2}$ is no more than an approximation to left ventricular ejection time and $M_{1} A_{1}$ is not a measure of isovolumic contraction time.

The method used to calculate the acceleration undergone by the ball assumes that this remains constant during the period of ball travel. If acceleration varies during this period, at some time it must be greater than the calculated value which therefore represents the minimum acceleration compatible 
with the observed displacement. Values for peak velocity have been derived directly from acceleration and are therefore subject to the same limitation. Nevertheless, there is excellent agreement with published results (Gimenez et al., 1965; Winters, Gimenez, and Soloff, 1967) for both acceleration ( $1 \cdot 8 \mathrm{~g})$ measured angiocardiographically and peak velocity $(23-57 \mathrm{~cm}$. $/ \mathrm{sec}$.) measured by an ultrasound method, suggesting that the acceleration may not vary greatly during the period of ball travel.

Increase in ball travel time is associated with a delayed opening sound and hence with prolongation of $\mathrm{QA}_{1}$. This interval consists of three components: left ventricular activation time, left ventricular isovolumic contraction time, and ball travel time. The first remains constant under a wide variety of conditions (Aygen and Braunwald, 1962) and in the present study this was assumed to be the case for all normally conducted beats. Though isovolumic contraction time and ball travel time vary with inotropic stimuli, it was possible to show in 6 patients that the greater part of any change in $\mathbf{Q A}_{1}$ occurring as a result of exercise, ectopic beats, or atrial fibrillation was due to a change in ball travel time. Measurement of $\mathrm{QA}_{1}$ has the advantage that only a phonocardiogram and electrocardiogram are required, and therefore frequent records can be made with little disturbance to the patient.

A decrease in ball travel time may be due to an increase in the force of ventricular contraction, or to a decrease in aortic impedance, which was measured as the difference between end-diastolic aortic pressure and end-diastolic wedge pressure, representing the gradient across the prosthesis at the onset of left ventricular contraction. This varies, not only with changes in aortic flow, as in exercise, but also with sudden changes in $R R$ interval: an increase in the length of diastole results in a lower end-diastolic aortic pressure, and a similar or slightly higher wedge (PC) pressure immediately before the succeeding beat (Fig. $8 b)$. Such changes in aortic impedance associated with ectopic beats are quite distinct from changes in left ventricular function resulting from interval effects which may depend on the relative rates of decay of positive and negative inotropic energy of activation (Koch-Weser and Blinks, 1963). Nevertheless, they have similar effects on ball acceleration, and an inotropic effect cannot be substantiated unless changes in end-diastolic gradient across the prosthesis have been excluded as a cause.

Exercise-induced tachycardia was accompanied by an increase in the end-diastolic gradient across the prosthesis and by a reduc- tion in ball travel time probably resulting from the action of a positive inotropic stimulus on the left ventricle. This was not the effect of tachycardia alone, since there was no change in $\mathrm{QA}_{1}$ when the heart rate increased after atropine administration, or with ventricular pacing; in addition, when the heart rate was held constant by pacing the positive inotropic effect could still be shown for 2-3 minutes after a period of exercise. Such a relation between heart rate and ball travel time is likely to be due to positive inotropic and chronotropic effects of increased sympathetic activity, whereas an increase in heart rate in the absence of any change in ball travel time must be ascribed to some other cause. Thus, in our patients, changes in heart rate at all exercise levels were mediated by changes in sympathetic tone. This conclusion differs from that of Robinson et al. (1966), who found that the initial increase in heart rate during supine exercise in fit young men was due to parasympathetic inhibition. This difference probably reflects the increased dependence of myocardial function on sympathetic activity in patients with a chronically diseased left ventricle.

Alterations in ventricular filling had little effect on $Q_{1}$, even when associated with changes in heart rate. Movement from supine to erect position, known to be associated with a reduction in stroke volume (Chapman, Fisher, and Sproule, 1960), caused no significant change in $\mathrm{QA}_{1}$ in Io patients: though stroke volume was not measured directly, the concomitant reduction in $A_{1} A_{2}$ suggests that it was in fact reduced.

Changes in ventricular filling cannot therefore be invoked to explain the increase in ball travel time associated with ectopic beats. This might have been due to the shorter RR interval immediately preceding, either as a direct rate effect, or associated with the increased end-diastolic gradient across the prosthesis. In addition, abnormal activation might lead to asynchronous ventricular contraction. Similarly, post-ectopic accentuation might also be related either to a rate effect, or to an abnormally low end-diastolic gradient across the prosthesis (Fig. 8b). The alternation of $\mathrm{QA}_{1}$ and of ball travel time for several beats after an ectopic beat suggests a relation with pulsus alternans (Fig. 8a).

It is likely that ball acceleration in our patients was related to the initial acceleration of blood into the ascending aorta. A negative pressure gradient can be shown across the normal aortic valve for the greater part of left ventricular ejection showing that movement of blood into the aorta is governed, not by 
pressure-flow relation, but by the determinants of mass acceleration (Spencer and Greiss, 1962; Noble, 1968). In our patients, forces generated by the left ventricle caused movement, not only of blood but also of the ball of the prosthesis, and it is therefore to be expected that the two would be closely related. The low mass $(2-3 g)$ and relatively large surface area of the ball make it unlikely that an appreciable velocity gradient develops between it and the surrounding blood until it reaches the apex of the cage. Factors controlling the peak acceleration of blood into the ascending aorta of the dog have been studied by a number of workers (Rushmer et al., 1963; Rushmer, 1964; Noble et al., 1966a, b, c). The resting value is somewhat higher than the ball acceleration measured in the present series of patients $(4.7 \mathrm{~g}-$ Spencer and Greiss, 1962; 5.I $g$ - Rushmer et al., 1963; $7.6 \mathrm{~g}-$ Noble et al., 1966a). Intracoronary injection of calcium or isoprenaline causes a conspicuous increase in peak acceleration while it is unaffected by changes in posture (Noble et al., 1966a) and reduced slightly when heart rate is increased by right atrial pacing (Noble et al., 1966c). It is also reduced by a sudden increase in aortic impedance (Wilcken et al., 1964). The over-all similarity to the present results supports a close relation between the initial acceleration of the ball and the peak acceleration of blood into the ascending aorta. This latter has proved to be a very sensitive measure of certain inotropic stimuli in experimental animals, and the present method provides a simple way of assessing a related variable in man.

Our thanks are due to Miss J. Woods and Mr. J. Castle for technical assistance, and to Mr. P. E. Chappel, Jr., of Edwards Laboratories Inc. for information about the dimensions of StarrEdwards prostheses.

\section{References}

Aygen, M. M., and Braunwald, E. (1962). The splitting of the second heart sound in normal subjects and in patients with congenital heart disease. Circulation, 25, 328.

Chapman, C. B., Fisher, J. N., and Sproule, B. J. (1960). Behavior of stroke volume at rest and during exercise in human beings. Fournal of Clinical Investigation, 39, 1208.

Dayem, M. K. A., and Raftery, E. B. (1967). Phonocardiogram of the ball-and-cage aortic valve prosthesis. British Heart fournal, 29, 446.

Franklin, D. L., Van Citters, R. L., and Rushmer, R. F. (1962). Left ventricular function described in physical terms. Circulation Research, 11, 702.

Gimenez, J. L., Winters, W. L., Davila, J. C., Connell, J., and Klein, K. S. (1965). Dynamics of the StarrEdwards ball valve prosthesis: A cinefluorographic and ultrasonic study in humans. American fournal of the Medical Sciences, 250, 652.

Koch-Weser, J., and Blinks, J. R. (1963). The influence of the interval between beats on myocardial contractility. Pharmacological Reviews, 15, 601.

Noble, M. I. M. (1968). The contribution of blood momentum to left ventricular ejection in the dog. Circulation Research, 23, 663.

—, Trenchard, D., and Guz, A. (1966a). Left ventricular ejection in conscious dogs. I. Measurement and significance of the maximum acceleration of blood from the left ventricle. Circulation Research, 19, 139.

,-- , and - (1966b). Left ventricular ejection in conscious dogs. 2. Determinants of stroke volume. Circulation Research, 19, 148.

,-- , and - (1966c). Effect of changing heart rate on cardiovascular function in the conscious dog. Circulation Research, 19, 206.

Robinson, B. F., Epstein, S. E., Beiser, G. D., and Braunwald, E. (1966). Control of heart rate by the autonomic nervous system. Studies in man on the interrelation between baroreceptor mechanisms and exercise. Circulation Research, 19, 400.

Rushmer, R. F. (1964). Initial ventricular impulse. A potential key to cardiac evaluation. Circulation, 29, 268.

- Watson, N., Harding, D., and Baker, D. (1963). Effects of acute coronary occlusion on performance of right and left ventricles in intact unanesthetized dogs. American Heart fournal, 66, 522.

Spencer, M. P., and Greiss, F. C. (1962). Dynamics of ventricular ejection. Circulation Research, 10, 274.

Wilcken, D. E. L., Charlier, A. A., Hoffman, J. I. E., and Guz, A. (1964). Effects of alterations in aortic impedance on the performance of the ventricles. Circulation Research, 14, 283.

Winters, W. L., Gimenez, J., and Soloff, L. A. (1967). Clinical application of ultrasound in the analysis of prosthetic ball valve function. American fournal of Cardiology, 19, 97. 